\title{
NUMERO DI INCIDENTI CAUSATI DA ANIMALI VELENOSI TRA IL 2010 E IL 2014 IN BRASILE
}

\section{ARTICOLO ORIGINALE}

SOEIRO, Selthon Macello Capiberibe ${ }^{1}$, FACCO, Lucas², FECURY, Amanda Alves ${ }^{3}$, ARAÚJO, Maria Helena Mendonça de ${ }^{4}$, OLIVEIRA, Euzébio de ${ }^{5}$, DENDASCK, Carla Viana $^{6}$, SOUZA, Keulle Oliveira da ${ }^{7}$, DIAS, Claudio Alberto Gellis de Mattos ${ }^{8}$

SOEIRO, Selthon Macello Capiberibe. Et. Numero di incidenti causati da animali velenosi tra il 2010 e il 2014 in Brasile. Revista Científica Multidisciplinar Núcleo do Conhecimento. Anno 05, Ed. 12, Vol. 01, pp. 05-17. dicembre 2020. ISSN: 24480959, Link di accesso: https://www.nucleodoconhecimento.com.br/salute/numero-diinfortuni, DOI: 10.32749/nucleodoconhecimento.com.br/salute/numero-di-infortuni

\section{RIEPILOGO}

Gli animali velenosi sono quelli che hanno un meccanismo per iniettare veleno. Diversi veleni o veleno possono causare dolore, rottura dei vasi sanguigni, ustioni, vesciche, edemi, lesioni necrotiche, emorragia, vomito nel sangue, presenza di sangue nelle urine, sanguinamento nel naso, sudorazione intensa, bassa pressione sanguigna, ipotermia e problemi neurologici. Lo scopo di questo articolo è quello di mostrare il numero di incidenti causati da animali velenosi tra il 2010 e il 2014 in Brasile. Considerate cause trascurate di malattie, gli incidenti causati da animali

\footnotetext{
${ }^{1}$ Laurea in corso in Informatica.

${ }^{2}$ Studente del Corso di Medicina dell'Università Federale di Amapá (UNIFAP).

${ }^{3}$ Biomedicale, Dottorato di Ricerca in Malattie Tropicali, Professore e ricercatore del Corso di Medicina dell'Università Federale di Amapá (UNIFAP).

${ }^{4}$ Medico, professore e ricercatore del corso di medicina dell'Università federale di Amapá (UNIFAP).

${ }^{5}$ Biologo, Dottore di Ricerca in Malattie Topiche, Professore e ricercatore del Corso di Educazione Fisica dell'Università Federale di Pará (UFPA).

${ }^{6}$ Teologo, Dottore di Ricerca in Psicoanalisi, ricercatore presso il Centro di Ricerca e Studi Avanzati - CEPA.

${ }^{7}$ Sociologo, studente magistrale in Studi Antropici in Amazzonia, Membro del Gruppo di Ricerca "Laboratorio di Educazione, Ambiente e Salute" (LEMAS/UFPA).

${ }^{8}$ Biologo, Dottore di Ricerca in Teoria e Comportamento, Professore e ricercatore del Graduate Program in Professional and Technological Education (PROFEPT), Istituto Federale di Amapá (IFAP).
}

RC: 67471

Disponibile in: https://www.nucleodoconhecimento.com.br/salute/numero-di-infortuni 
velenosi richiedono una maggiore attenzione per la salute pubblica. Ragni, scorpioni e serpenti sono i principali causari di questi incidenti. Tra i bambini, i ragazzi sono i più colpiti e la mancanza di supervisione nelle attività quotidiane è una delle principali cause di questo problema. La produzione di siero per il trattamento degli incidenti odidisi in Brasile è accentuata e la quantità prodotta è in grado di coprire il numero totale di casi nel paese. Tuttavia, esiste un problema per quanto riguarda la distribuzione di questo siero, poiché le aree della popolazione indigena e le regioni di località remote non hanno un'adeguata disponibilità, consentendo conseguenze negative per gli individui in questione, aumentando la possibilità di decessi per questo tipo di incidenti.

Parole chiave: Epidemiologia, veleno, veleno.

\section{INTRODUZIONE}

Gli animali velenosi sono quelli che hanno un meccanismo per iniettare veleno, che può essere pungiglioni, pungiglioni o denti (Pinho e Pardal, 2015). Alcune specie di api, serpenti, vespe, calabroni, ragni, scorpioni e bruchi possono essere considerate animali velenosi (BREDT e LITCHTENEKER, 2014; NEIVA, et al., 2019).

Diversi veleni o veleno possono causare dolore, rottura dei vasi sanguigni, ustioni, vesciche, edemi, lesioni necrotiche, emorragia, vomito nel sangue, presenza di sangue nelle urine, sanguinamento nel naso, sudorazione grave, bassa pressione sanguigna, ipotermia e problemi neurologici (LIMA et al., 2017; NEIVA, et al., 2019).

II trattamento degli incidenti con animali velenosi viene effettuato, nella maggior parte dei casi, per mezzo di un siero antivenom che contiene anticorpi specifici per combattere le tossine di ogni animale, e questo dovrebbe essere applicato il prima possibile per evitare l'aggravamento del caso (SOUZA e MACHADO, 2017).

Per prevenire incidenti con serpenti, guanti e stivali dovrebbero essere utilizzati durante la pulizia di cespugli, detriti, foglie secche, spazzatura e altri, poiché tali

RC: 67471

Disponibile in: https://www.nucleodoconhecimento.com.br/salute/numero-di-infortuni 
animali preferiscono luoghi caldi e umidi. Non dovresti mettere le mani nei buchi, dovresti evitare la proliferazione dei ratti, poiché questi sono cibo serpente e, inoltre, è necessario evitare l'accumulo di cespuglio, legno, mattoni e altri materiali che possono diventare un rifugio per gli animali che fungono da cibo per i serpenti (BRASIL, 2001).

Per prevenire incidenti con aracnidi, terre desolate, giardini e cortili dovrebbero essere tenerli liberi da macerie, cespugli, foglie secche e materiali da costruzione, impedendo loro di trovare riparo. Inoltre, si dovrebbe: evitare le piante con fogliame denso vicino alle pareti; scuotere vestiti e scarpe prima di indossare; indossare guanti e scarpe durante la manipolazione e la camminata in luoghi che possono contenere tali animali; aperture di tenuta dove gli aracnidi possono attraversare (principalmente di notte, a causa delle loro abitudini); mettere gli schermi in scarichi, lavandini e serbatoi; prevenire la proliferazione di insetti che sono il cibo degli aracnidi (una delle misure è mantenere i rifiuti domestici al chiuso per evitare scarafaggi e mosche); rimuovere i letti dalle pareti e non lasciare che moschettieri e lenzuola tocchino il pavimento; evitare di uccidere predatori aracnidi (rane, uccelli, lucertole, polli, oche, scimmie e altri) (BRASIL, 2001).

II numero di casi in Brasile tra il 1986 e il 2016 è stato di 2.183.989. II numero di casi per regione in Brasile, tra il 1986 e il 2016, sono: regione nord: 233.586; Regione nord-orientale: 624.615; Regione sud-orientale: 758.238; Regione sud: 427.442; Regione del Midwest: 140.108. II numero di casi ad Amapá tra il 1986 e il 2016 in Amapá 8463 (BRASIL, 2017).

\section{OBIETTIVI}

Mostra il numero di incidenti causati da animali velenosi tra il 2010 e il 2014 in Brasile.

RC: 67471

Disponibile in: https://www.nucleodoconhecimento.com.br/salute/numero-di-infortuni 


\section{METODO}

La ricerca è stata condotta nel database DATASUS (http://datasus.saude.gov.br/). I dati nazionali sono stati raccolti secondo i seguenti passaggi: A) Abbiamo effettuato l'accesso al collegamento datasus.saude.gov.br, spostato la freccia con il mouse sulla scheda "Accesso alle informazioni", quindi siamo andati alla scheda "Informazioni sanitarie (TABNET) )", cliccato su "Epidemiologia e morbilità". Nella pagina successiva, cliccare su "Malattie e disturbi notificati - Dal 2007 in poi (SINAN)". Nella pagina successiva abbiamo selezionato "Accidents by Poisonous Animals. Nella casella "Ambito geografico" l'opzione era "Brasile per Regione, Stato e Comune". Da quel momento in poi sono stati seguiti i passaggi: A) Sulla riga è stato selezionato "Anno incidente"; nella colonna "Non attivo" e nel contenuto "Notifiche". Tutti i dati raccolti in questa e nelle prossime fasi sono compresi tra il 2010 e il 2014. B) Nella riga è stato selezionato "Anno incidente"; nella colonna "Mese dell'incidente" e nel contenuto "Notifiche". C) Nella riga è stato selezionato "Anno incidente"; nella colonna "Regione di notifica" e nel contenuto "Notifiche". D) Sulla riga è stato selezionato "Anno incidente"; nella colonna "Fasce di età", e nel contenuto "Notifiche". E) Nella riga è stato selezionato "Anno incidente"; nella colonna "Gara", e nel contenuto "Notifiche". F) Nella riga è stato selezionato "Anno incidente"; nella colonna "Sesso" e nel contenuto "Notifiche". G) Sulla riga è stato selezionato "Anno incidente"; nella colonna "Donna incinta" e nel contenuto "Notifiche". H) Sulla riga è stato selezionato "Anno incidente"; nella colonna "Riduzione orari/servizio", e nel contenuto "Notifiche". I) Nella riga è stato selezionato "Anno incidente"; nella colonna "Tipo di sinistro" e nel contenuto "Notifiche". J) Nella riga è stato selezionato "Anno incidente"; nella colonna "Tariffa. Finale", e nel contenuto "Notifiche". K) Sulla riga è stato selezionato "Anno incidente"; nella colonna "Evoluzione del caso", e nel contenuto "Notifiche"

I dati sono stati compilati all'interno dell'applicazione Excel, un componente della suite Microsoft Corporation Office. La ricerca bibliografica è stata condotta in articoli scientifici, utilizzando computer del laboratorio informatico dell'Istituto Federale di

$\mathrm{RC}: 67471$

Disponibile in: https://www.nucleodoconhecimento.com.br/salute/numero-di-infortuni 
Educazione, Scienza e Tecnologia di Amapá, Macapá Campus, situato a: Rodovia BR 210 KM 3, s/n - Bairro Brasil Novo. CAP: 68.909-398, Macapá, Amapá, Brasile.

\section{RISULTATI}

La figura 1 mostra il numero di incidenti causati da animali velenosi tra il 2010 e il 2014, indicando un aumento ogni anno.

La figura 1 mostra il numero di casi di incidenti causati da animali velenosi in Brasile tra il 2010 e il 2014.

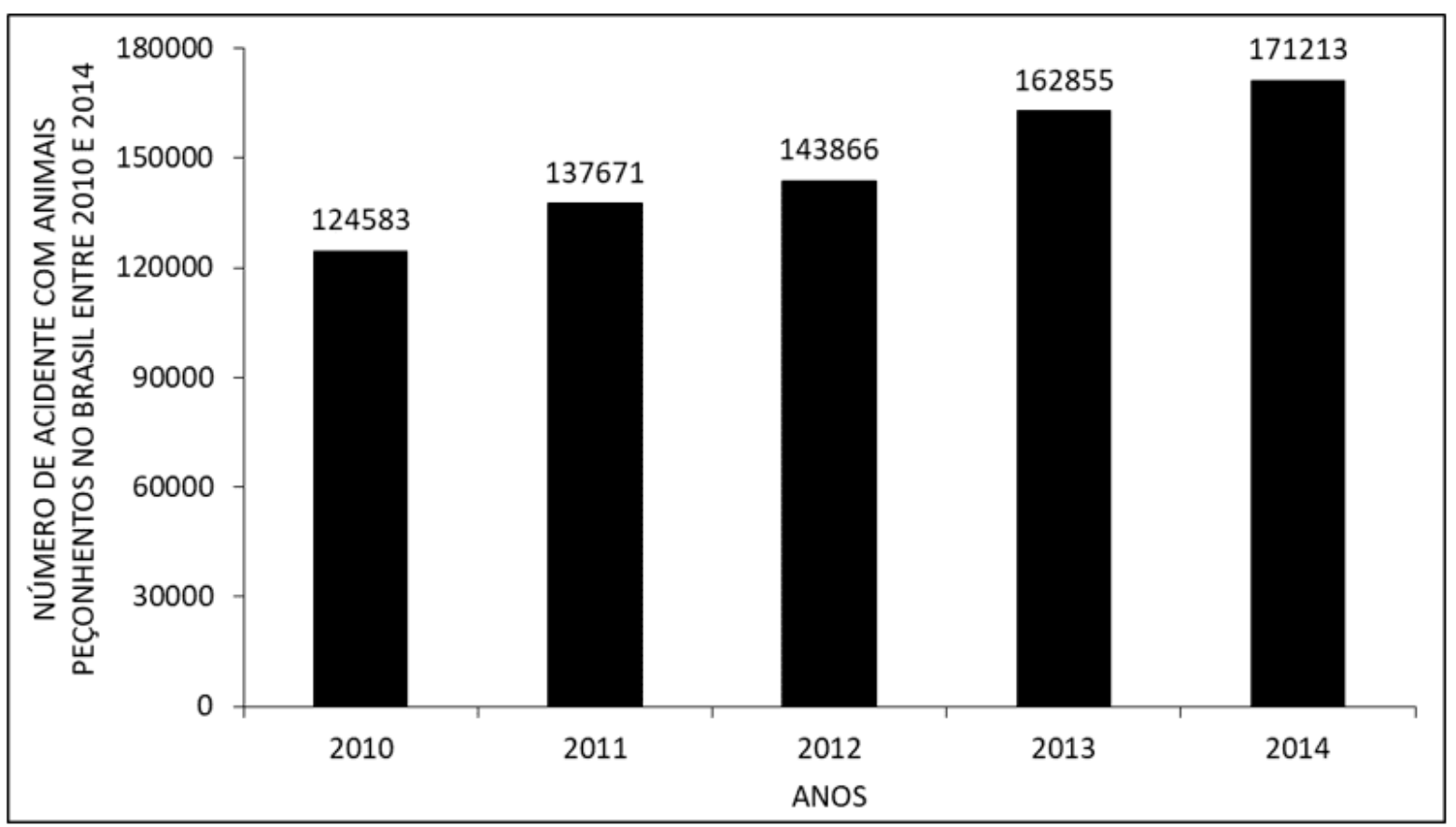

La figura 2 mostra il numero di incidenti causati da animali velenosi al mese dell'anno tra il 2010 e il 2014. II numero maggiore si verifica a gennaio, con un calo a febbraio, in aumento ancora a marzo e di nuovo in calo fino a luglio. Questo è il mese con il minor numero di casi, con un aumento nei mesi successivi.

$\mathrm{RC}: 67471$

Disponibile in: https://www.nucleodoconhecimento.com.br/salute/numero-di-infortuni 
La figura 2 mostra il numero di casi di incidenti causati da animali velenosi in Brasile tra il 2010 e il 2014, per mesi dell'anno.



La figura 3 mostra il numero di segnalazioni di incidenti causati da animali velenosi in Brasile tra il 2010 e il 2014, per regioni del paese. II maggior numero di casi si verifica nella regione sud-orientale seguita, rispettivamente, dalle regioni nord-est, sud, nord e midwest.

RC: 67471

Disponibile in: https://www.nucleodoconhecimento.com.br/salute/numero-di-infortuni 
La figura 3 mostra il numero di casi di incidenti causati da animali velenosi in Brasile tra il 2010 e il 2014, per regioni del paese.

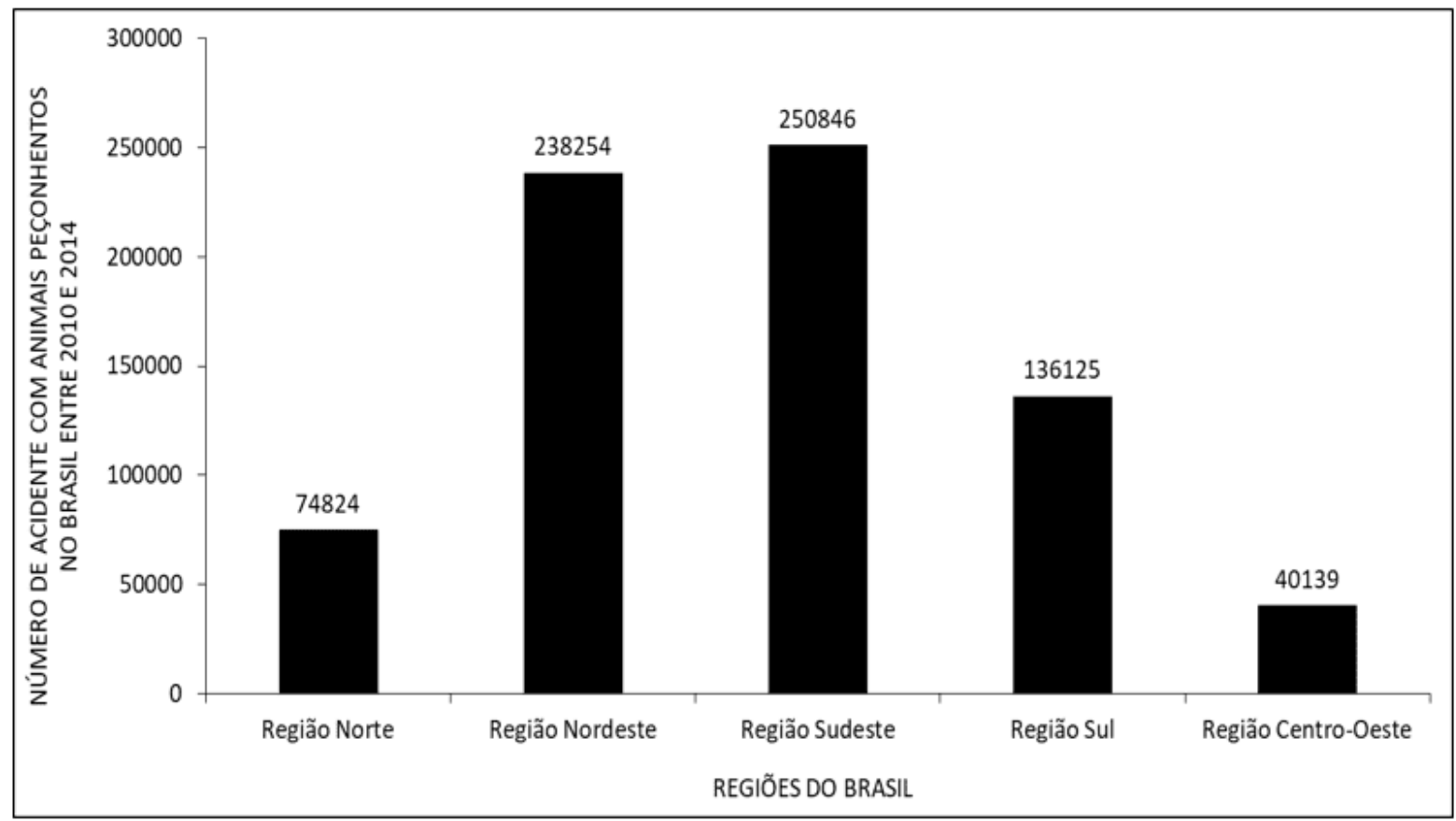

La figura 4 mostra il numero di segnalazioni di incidenti causati da animali velenosi in Brasile tra il 2010 e il 2014, per genere. II maggior numero di casi capita agli uomini. 
La figura 4 mostra il numero di casi di incidenti causati da animali velenosi in Brasile tra il 2010 e il 2014, per genere.

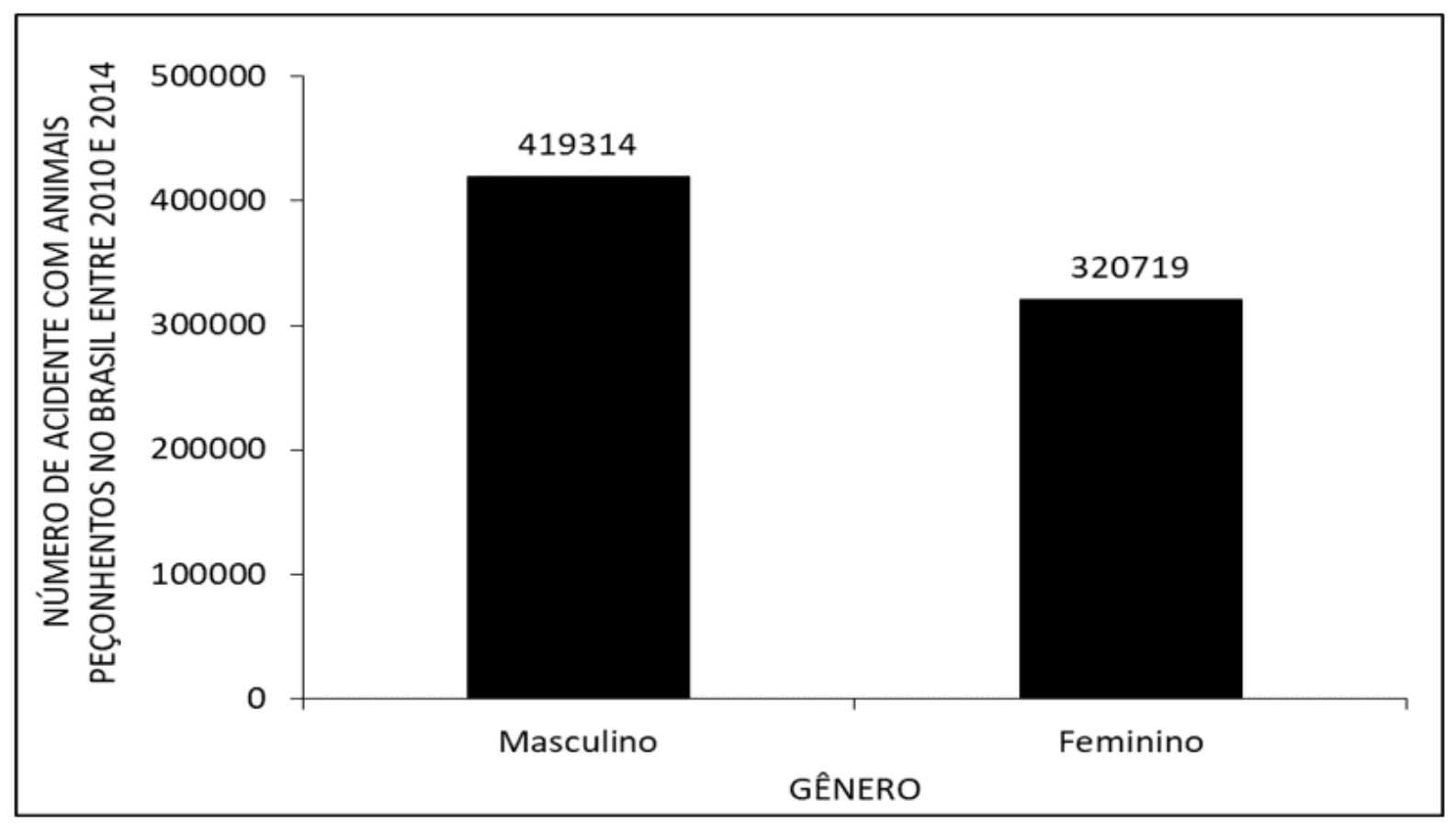

La figura 5 mostra il numero di segnalazioni di incidenti causati da animali velenosi in Brasile tra il 2010 e il 2014, per fascia d'età. II maggior numero di casi, secondo i dati, si verifica con persone tra i 20 e i 39 anni. II secondo maggior numero di casi figura nel gruppo tra i 40 e i 59 anni.

RC: 67471

Disponibile in: https://www.nucleodoconhecimento.com.br/salute/numero-di-infortuni 
La figura 5 mostra il numero di casi di incidenti causati da animali velenosi in Brasile tra il 2010 e il 2014, per fascia d'età.

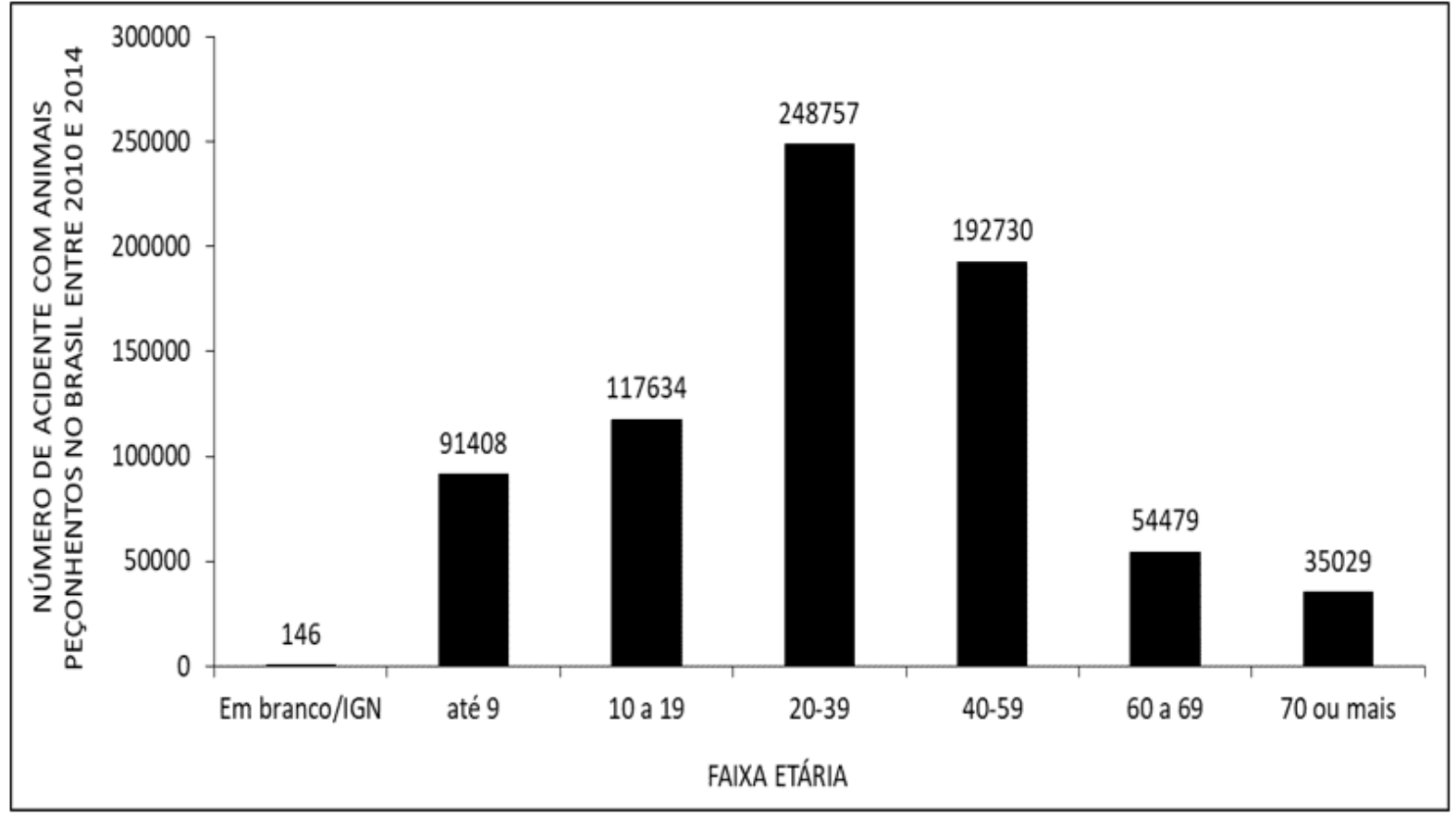

La figura 6 mostra il numero di segnalazioni di incidenti causati da animali velenosi in Brasile tra il 2010 e il 2014, per etnia. II maggior numero di casi si verifica con persone di etnia bruna, seguite rispettivamente da persone dichiarate persone bianche e nere.

RC: 67471

Disponibile in: https://www.nucleodoconhecimento.com.br/salute/numero-di-infortuni 
La figura 6 mostra il numero di casi di incidenti causati da animali velenosi in Brasile tra il 2010 e il 2014 , per etnia.

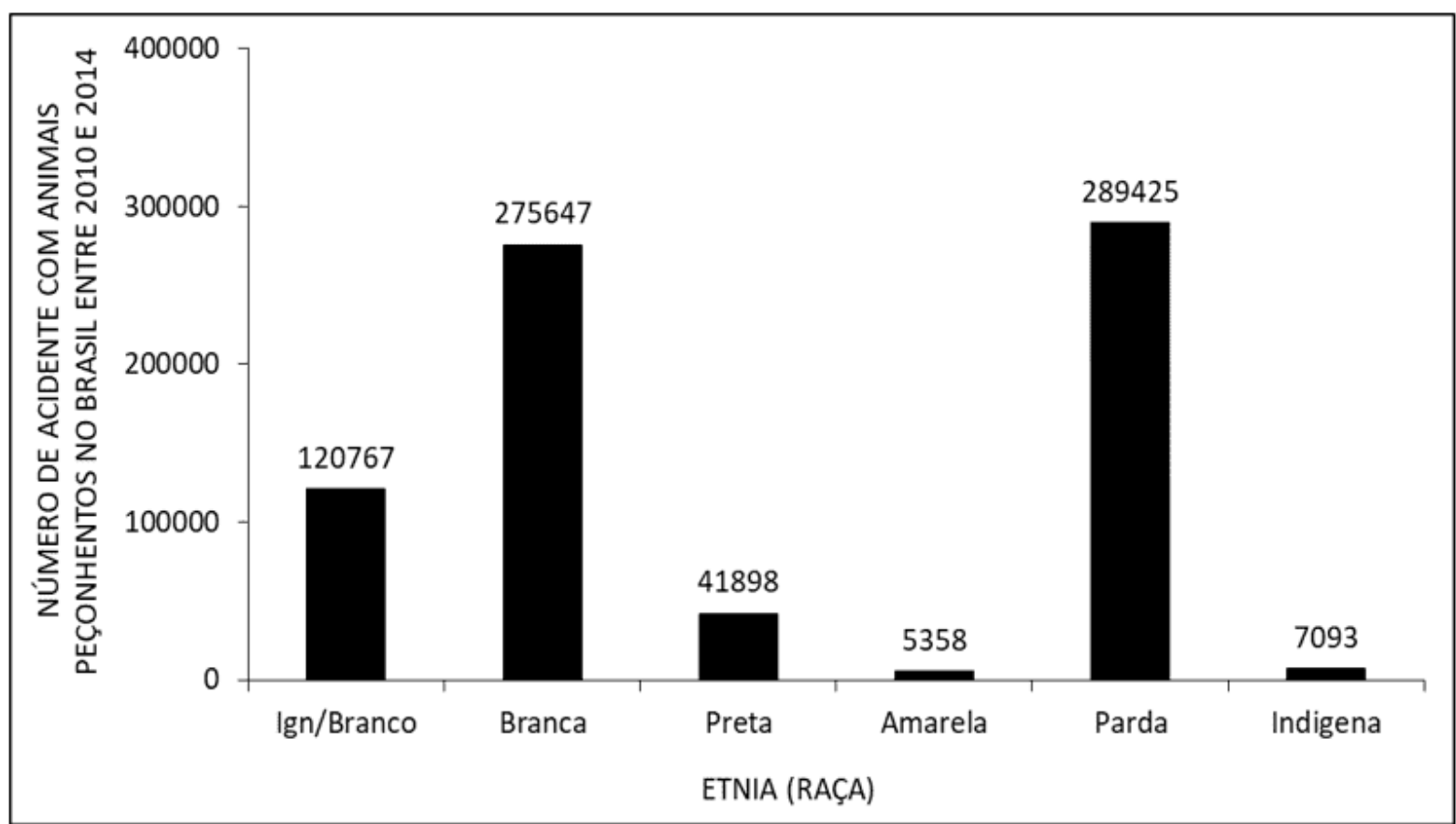

La figura 7 mostra il numero di segnalazioni di incidenti causati da animali velenosi in Brasile tra il 2010 e il 2014, per tipo di incidente. II maggior numero di casi si è verificato in incidenti che coinvolgono scorpioni.

RC: 67471

Disponibile in: https://www.nucleodoconhecimento.com.br/salute/numero-di-infortuni 
La figura 7 mostra il numero di casi di incidenti causati da animali velenosi in Brasile tra il 2010 e il 2014, per tipo di incidente.

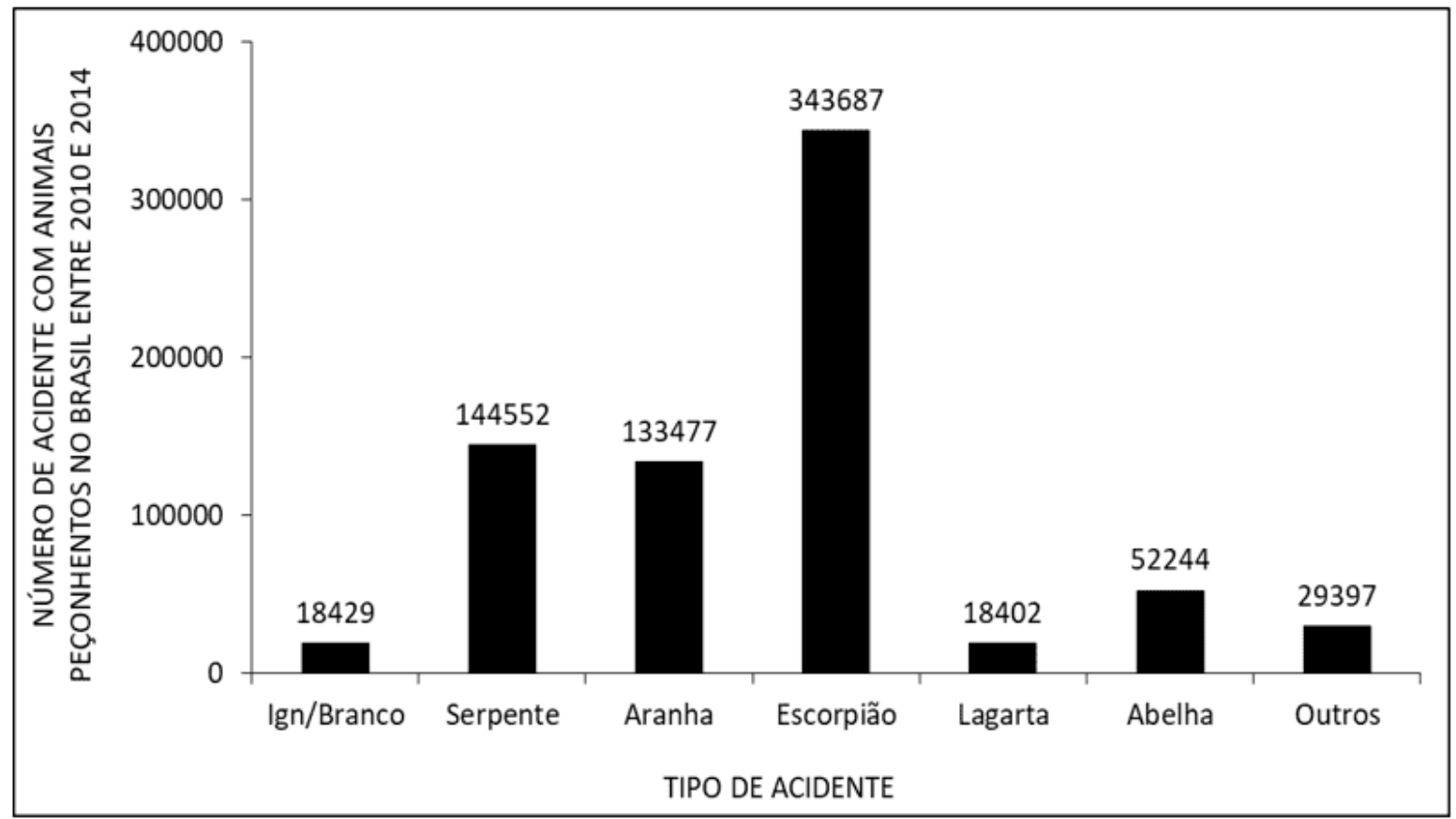

La figura 8 mostra il numero di segnalazioni di incidenti causati da animali velenosi in Brasile tra il 2010 e il 2014, a seconda delle fasi della gravidanza. II maggior numero di casi si verifica con donne incinte nel secondo trimestre, seguite da donne incinte rispettivamente nel primo e nel terzo trimestre.

RC: 67471

Disponibile in: https://www.nucleodoconhecimento.com.br/salute/numero-di-infortuni 
La figura 8 mostra il numero di casi di incidenti causati da animali velenosi in Brasile tra il 2010 e il 2014, in base alle fasi della gravidanza.

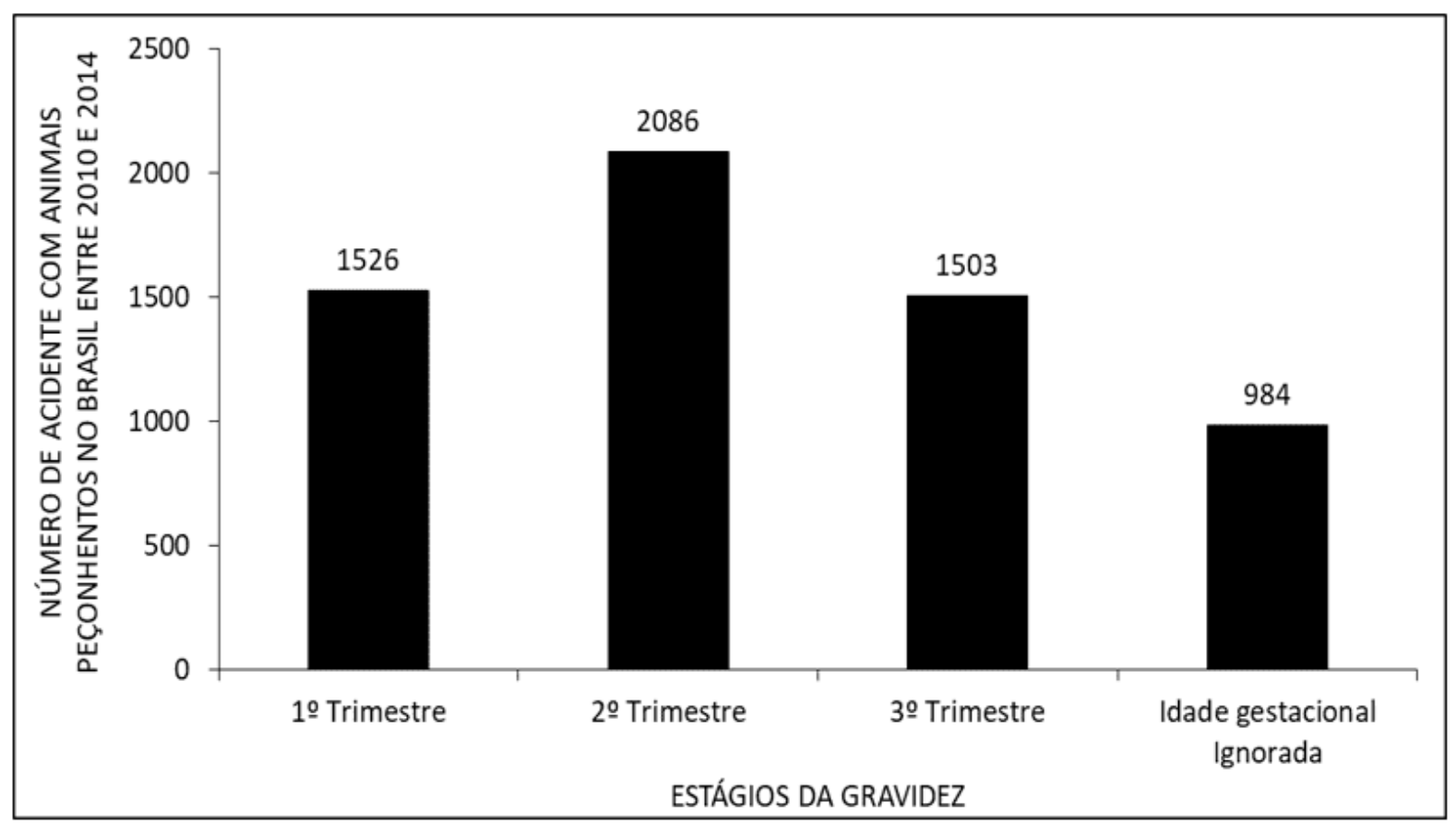

La figura 9 mostra il numero di segnalazioni di incidenti causati da animali velenosi in Brasile tra il 2010 e il 2014, in base all'ora in ore tra il morso e il servizio. I dati mostrano che il maggior numero di persone è frequentato tra 0 e 3 ore dopo l'incidente.

RC: 67471

Disponibile in: https://www.nucleodoconhecimento.com.br/salute/numero-di-infortuni 
La figura 9 mostra il numero di casi di incidenti causati da animali velenosi in Brasile tra il 2010 e il 2014, in base al tempo in ore tra il morso e la cura.

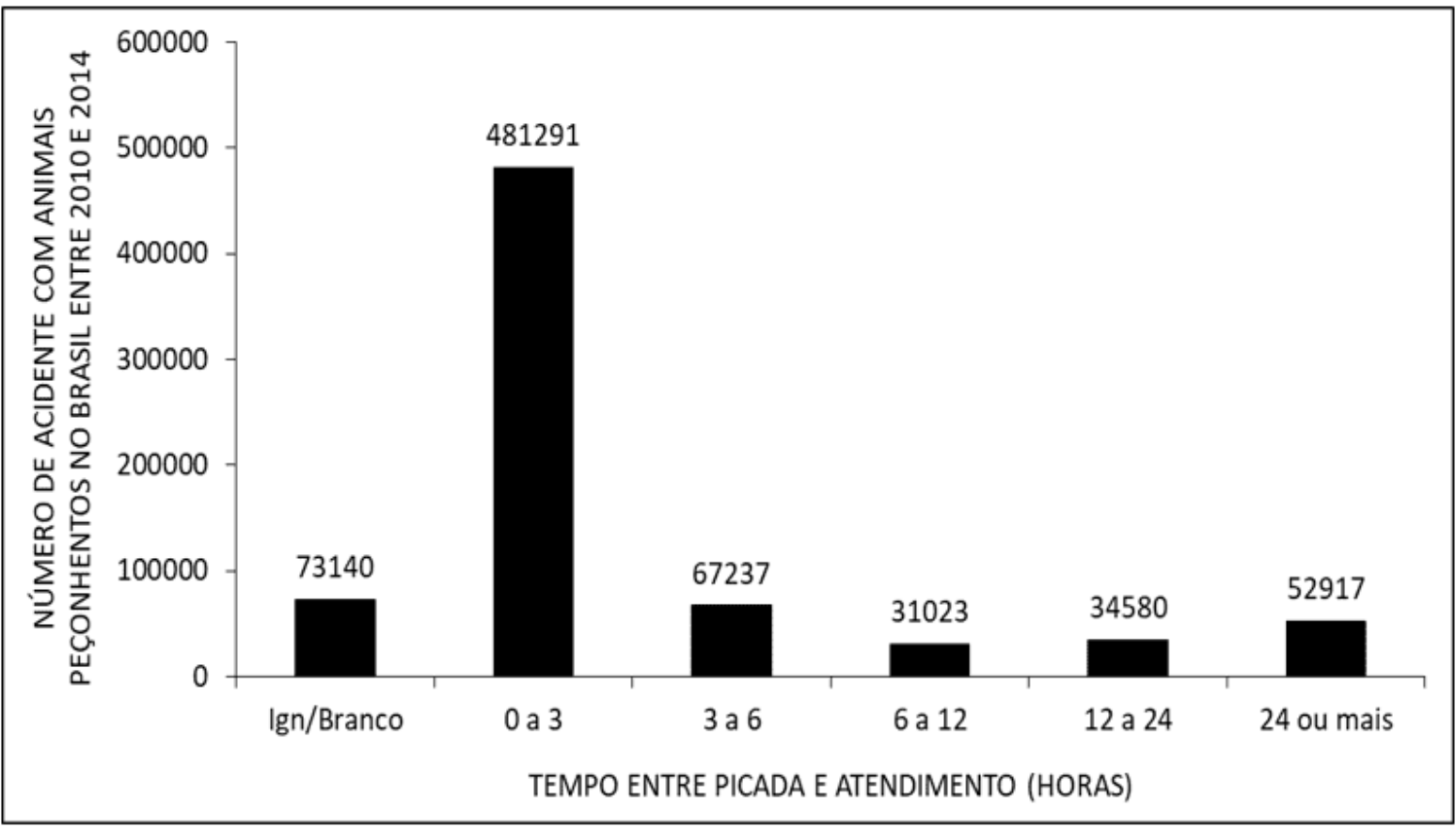

La figura 10 mostra il numero di segnalazioni di incidenti causati da animali velenosi in Brasile tra il 2010 e il 2014, secondo la classificazione finale dell'incidente. II maggior numero di casi ha avuto come classificazione finale dell'infortunio di grado lieve.

RC: 67471

Disponibile in: https://www.nucleodoconhecimento.com.br/salute/numero-di-infortuni 
Figura 10 Mostra il numero di casi di incidenti causati da animali velenosi in Brasile tra il 2010 e il 2014, per classificazione finale dell'incidente.

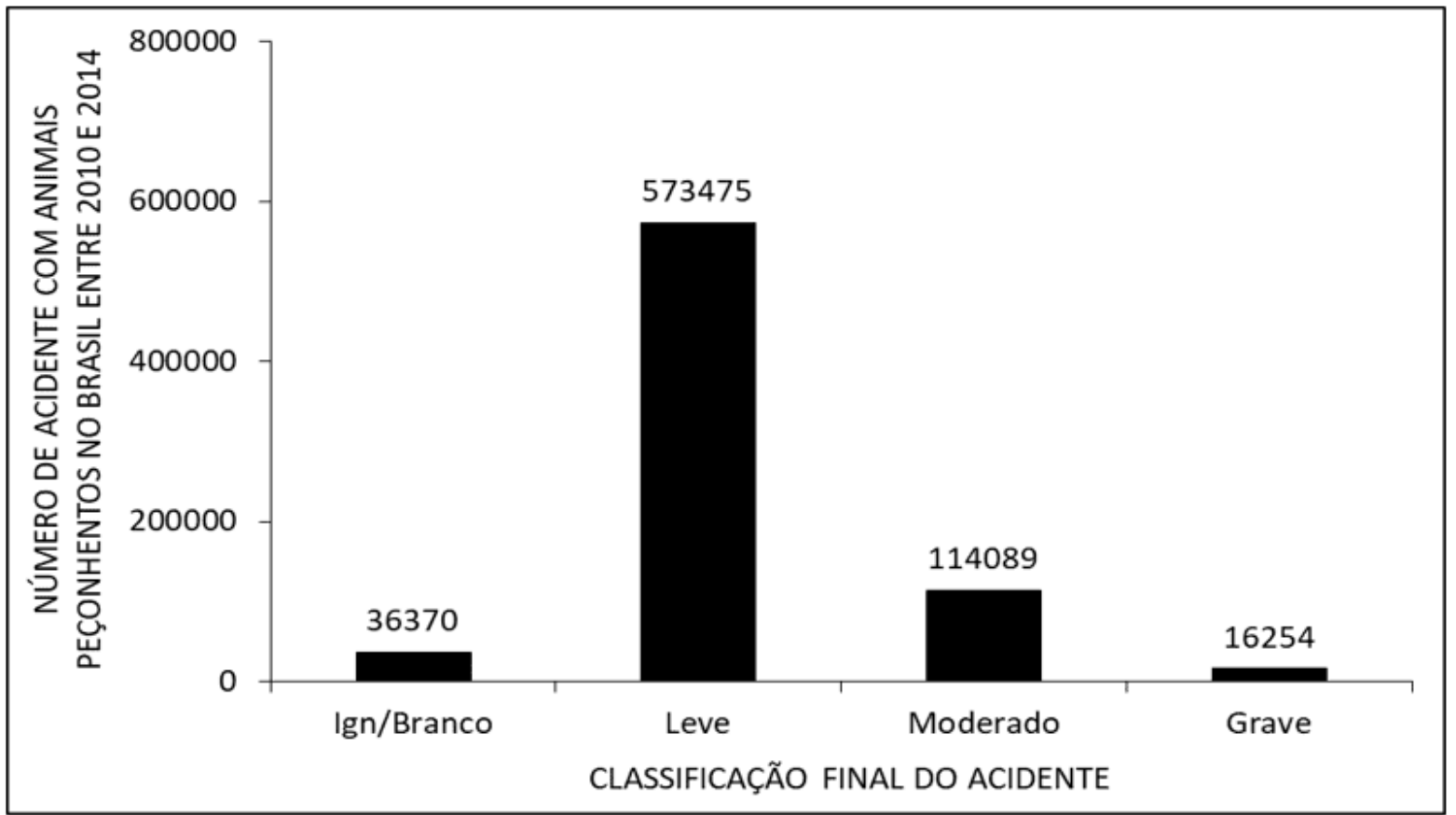

La figura 11 mostra il numero di segnalazioni di incidenti causati da animali velenosi in Brasile tra il 2010 e il 2014, per evoluzione del caso. II maggior numero di casi è stato curato, con un importo minimo che è morto.

RC: 67471

Disponibile in: https://www.nucleodoconhecimento.com.br/salute/numero-di-infortuni 
La figura 11 mostra il numero di casi di incidenti causati da animali velenosi in Brasile tra il 2010 e il 2014, a causa dell'evoluzione del caso.

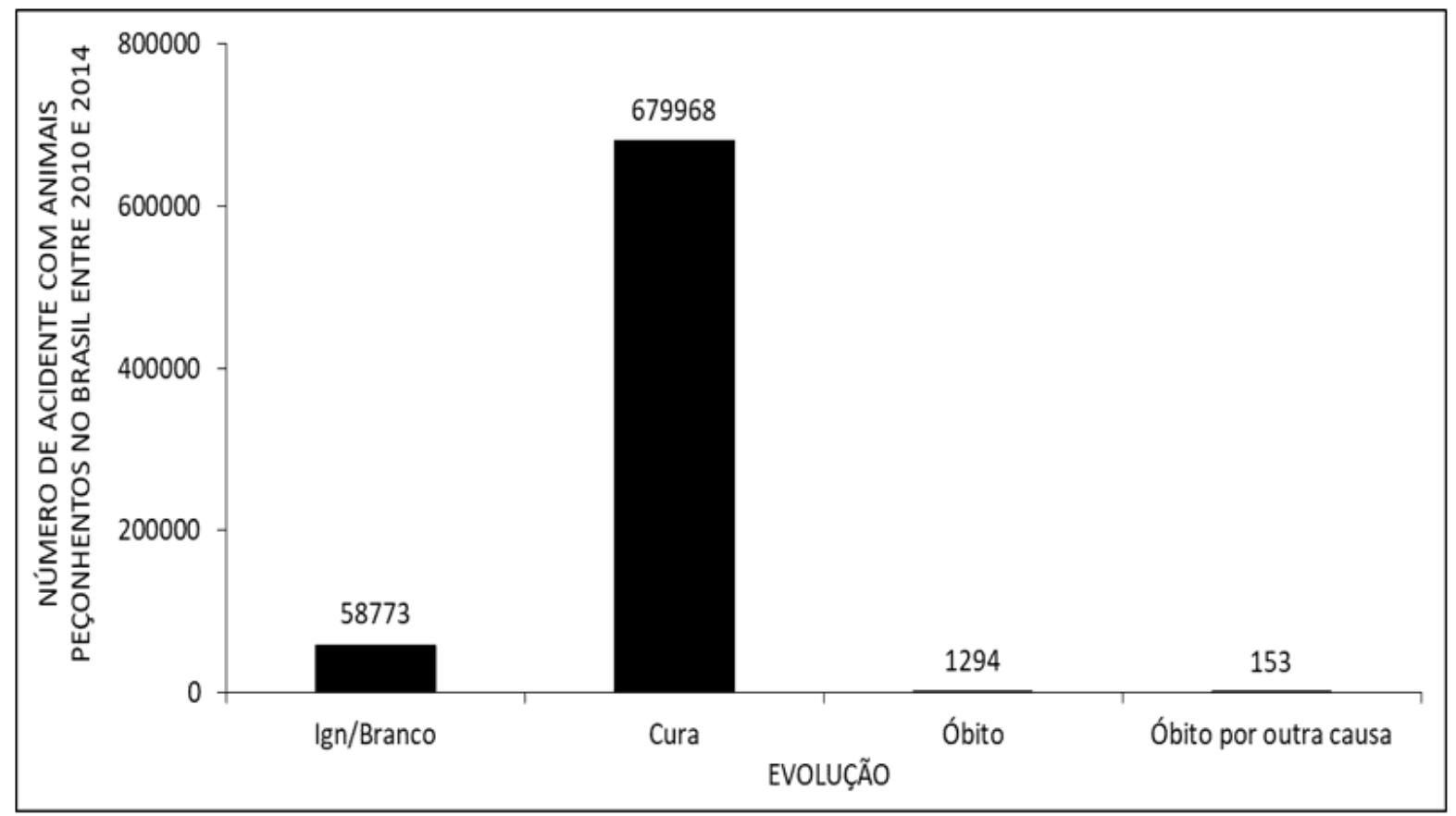

\section{DISCUSSIONE}

Gli incidenti causati da animali velenosi sono considerati cause trascurate di malattie e il loro verificarsi è più elevato nelle zone rurali e nei paesi la cui povertà è diffusa. In grado di generare una serie di perdite, tali incidenti sono in grado di provocare conseguenze a livello organico e socioeconomico, poiché giustificano la necessità di investimenti in metodi profilattici e sieri per il trattamento di tali partecipazioni (SALOMÃO et al., 2018). Considerati come un'emergenza clinica (cioè, in cui è necessaria un'attenzione immediata al paziente), sono causati da una serie di animali velenosi, essendo scorpioni, ragni e serpenti. Nel 2013, in Brasile, ci sono stati 158.002 casi di incidenti con anima velenose, la maggior parte (circa il $78 \%$ del totale) causata da serpenti (SILVA et al., 2015). Gli infortuni sul lavoro che comportano cause basate su animali velenosi non sono riportati in modo ottimale e sono sottosegnala. Tuttavia, si è registrato un aumento in tali casi, tenendo conto di un periodo che copre dal 2007 al 2017 (BRASIL, 2019).

RC: 67471

Disponibile in: https://www.nucleodoconhecimento.com.br/salute/numero-di-infortuni 
La regione sud-orientale è il campione nel numero di incidenti causati da animali velenosi, con serpenti e scorpioni che sono le principali cause di tali eventi. Tra questi, gli scorpioni hanno dimostrato di essere le principali cause di questo problema, poiché trovano nelle aree urbane un buon adattamento all'ambiente, un fattore che fornisce riproduzione e conseguente proliferazione di questo animale nella regione. Gran parte degli incidenti con lo scorpione ha lievi conseguenze per l'organismo umano e non richiede l'uso della sieroterapia. Tuttavia, negli altri casi, può esserci un'ampia variazione nelle conseguenze da individuo a individuo, con la morte che è un possibile risultato se non esiste un trattamento adeguato. Inoltre, possono esserci sequele di tali incidenti, al fine di compromettere la dinamica quotidiana dell'individuo, rendendo difficile anche il lavoro (LIMA et al., 2020).

Gran parte dei casi di incidenti con animali velenosi (la maggior parte di essi) si verificano con individui maschi, e questa affermazione è valida per la stragrande maggioranza degli animali velenosi. In particolare, tra gli incidenti ofidici, il profilo epidemiologico più comune è quello dei lavoratori rurali, la cui età è compresa tra i 15 e i 49 anni (BRASIL, 2019). I ragazzi tendono a subire più incidenti da parte di animali velenosi in considerazione di aspetti quali la mancanza di supervisione nelle attività ricreative all'aperto, oltre ad altre questioni socioculturali (NEIVA et al., 2019). La fascia d'età che comprende il maggior numero di casi va da 20 a 39 anni e tra il 2009 e il 2013 si è registrato un aumento dei casi in questo gruppo (SILVA et al., 2015a). Alla luce dei dati raccolti, il maggior numero di individui colpiti da incidenti con animali velenosi è di etnia bruna, seguita da bianchi e neri.

Tra gli incidenti e i decessi causati da animali velenosi, gli scorpioni sono i più responsabili nel periodo in esame. Nella regione sudorientale (una regione in cui vi è una maggiore predominanza di incidenti da parte di animali velenosi), le cause dello scorpione sono responsabili del maggior numero medio di morti e incidenti in questa categoria (LIMA et al., 2020). Tra gli infortuni sul lavoro che si verificano a causa di cause dello scorpione, le parti del corpo più colpite sono le mani, i piedi e le braccia, tenendo conto del periodo dal 2007 al 2017 (BRASIL, 2019).

$\mathrm{RC}: 67471$

Disponibile in: https://www.nucleodoconhecimento.com.br/salute/numero-di-infortuni 
Gli incidenti botropici corrispondono all'ofidismo più comune nel paese, rappresentando circa il $90 \%$ del totale. Il veleno ha una serie di azioni a livello organico nell'individuo, essendo in grado di causare effetti proteolitici, coagulanti ed emorragici. Inoltre, dolore, edema, lividi e sanguinamento nella regione del morso sono comuni. Ci può anche essere la comparsa di vesciche e l'infarto di gruppi gangliari (può verificarsi necrosi della regione, ma non è sempre presente). Nelle donne in gravidanza, oltre alle manifestazioni in questione, c'è il rischio di emorragia uterina, caratterizzando un caso di maggiore gravità (BRASIL, 2001).

Secondo i dati presentati, tra le persone che hanno subito un incidente causato da animali velenosi, la maggior parte è frequentata dai servizi sanitari entro 3 ore dall'incidente. II tempo tra il morso e la cura dovrebbe essere il più piccolo possibile, e secondo i dati relativi allo stato di Rio Grande do Sul, con l'aumentare del tempo per le cure, aumenta la possibilità di conseguenze negative per l'individuo, come una diminuzione del numero di pazienti curati e una maggiore letalità. Sebbene il maggior numero di decessi si verifichi tra i maschi, le femmine hanno un tasso di letalità più elevato. Nel caso di pazienti anziani di età pari o superiore a 80 anni, è stato osservato un tasso di letalità più elevato rispetto ad altre fasce d'età (DORNELES, 2009).

Gli incidenti di animali velenosi, nella loro massima quantità, non rappresentavano una classificazione basata su uno stato di gravità, vale a dire che la maggior parte erano classificati come di grado lieve. II più alto tasso di gravità e letalità di solito si verifica nei bambini più grandi e negli individui (più di 80 anni) (DORNELES, 2009). In relazione agli incidenti con lo scorpione, la maggior parte può essere trattata in un'unità sanitaria situata vicino al paziente, senza richiedere sieroterapia (LIMA et al., 2020).

II Brasile è uno dei paesi che producono la sera più antivenom al mondo (circa 400.000 fiale). A livello nazionale, il trattamento è fornito dal Sistema Sanitario Unificato, essendo il Brasile il paese la cui distribuzione gratuita di siero antiofilo è resa più completa e con maggiore facilità, poiché è disponibile per eventuali feriti. La

$\mathrm{RC}: 67471$

Disponibile in: https://www.nucleodoconhecimento.com.br/salute/numero-di-infortuni 
quantità prodotta a livello nazionale è corretta in modo che possa servire il $100 \%$ degli individui brasiliani. Tuttavia, vi sono ancora complicazioni che devono essere risolte in relazione all'accesso completo a tutte le regioni, con aree indigene e regioni remote locali con minore disponibilità di siero (CUNHA, 2017).

\section{CONCLUSIONE}

Considerate cause trascurate di malattie, gli incidenti causati da animali velenosi richiedono una maggiore attenzione per la salute pubblica ed è necessario migliorare il sistema di notifica dei casi e una più ampia disponibilità di informazioni sull'argomento alla popolazione in generale. Ragni, scorpioni e serpenti sono i principali causari di questi incidenti.

II profilo epidemiologico più frequente negli incidenti odiosi sono gli uomini, i lavoratori rurali, tra i 15 e i 49 anni. Tra i bambini, i ragazzi sono i più colpiti e la mancanza di supervisione nelle attività quotidiane è una delle principali cause di questo problema.

La maggior parte degli incidenti dello scorpione genera conseguenze minori per l'organismo umano e non implica la necessità di sieroterapia. Negli altri casi, tuttavia, può esserci un'ampia variazione nelle conseguenze dal paziente al paziente e, se non esiste un trattamento adeguato, il paziente potrebbe morire.

La maggior parte dei casi è solitamente frequentata dai servizi sanitari entro 3 ore dall'infortunio. II tempo tra l'incidente e l'assistenza dovrebbe essere il più piccolo possibile e, con l'aumentare del tempo di cura, la possibilità di conseguenze negative per l'individuo aumenta proporzionalmente, con una diminuzione del tasso di cura e una maggiore letalità.

La maggior parte dei pazienti che hanno incidenti con scorpione non richiedono la sieroterapia e possono ricorrere a un trattamento appropriato in unità sanitarie che si

RC: 67471

Disponibile in: https://www.nucleodoconhecimento.com.br/salute/numero-di-infortuni 
trovano nelle vicinanze del luogo in cui si è verificato l'incidente, in modo che la locomozione sia più veloce e il conseguente tasso di miglioramento sia più alto.

La produzione di siero per il trattamento degli incidenti odidisi in Brasile è accentuata e la quantità prodotta è in grado di coprire il numero totale di casi nel paese. Tuttavia, esiste un problema per quanto riguarda la distribuzione di questo siero, poiché le aree della popolazione indigena e le regioni di località remote non hanno un'adeguata disponibilità, consentendo conseguenze negative per gli individui in questione, aumentando la possibilità di decessi per questo tipo di incidenti.

\section{RIFERIMENTI}

BRASIL, Fundação Nacional de Saúde. Manual de Diagnóstico e tratamento de acidentes por animais peçonhentos, $2^{\underline{a}}$ ed., Brasília, 2001.

BRASIL, Portal da saúde. Série Histórica de Casos - Acidente Por Animais Peçonhentos. 2017. Acessado em 26 de setembro de 2017<http://portalarquivos.saude.gov.br/images/pdf/2017/abril/28/1-

SerieHistorica_1986-2016.pdf>

BRASIL. Secretaria de Vigilância em Saúde. Ministério da Saúde. Boletim Epidemiológico 11: Acidentes de trabalho por animais peçonhentos entre trabalhadores do campo, floresta, águas, Brasil 2007 a 2017, v. 50, 2019.

BREDT C. S, LITCHTENEKER K. Avaliação Clínica e Epidemiológica dos acidentes com animais peçonhentos atendidos no Hospital Universitário do Oeste do Paraná 2008- 2012. Rev. Med. Res., Curitiba, v.16, n.1, p. 11-17, jan./mar. 2014.

CARMO É. A, NERY A. A, JESUS C. S, CASOTTI C. A. Internaçoes hospitalares por causas externas envolvendo contato com animais em um hospital geral do interior da Bahia, 2009-2011. Epidemiol. Serv. Saude, Brasília, p.105-114, jan./mar. 2016.

RC: 67471

Disponibile in: https://www.nucleodoconhecimento.com.br/salute/numero-di-infortuni 
CUNHA, L. E. R. Soros antiofídicos: história, evolução e futuro. Journal Health NPEPS, v. 2, supl. 1, p. 1-4, 2017.

DORNELES, A. L. Frequência de acidentes por animais peçonhentos ocorridos no Rio Grande do Sul, 2001 - 2006. Trabalho de Conclusão de curso, Especialização em Saúde Pública, Proto Alegre, RS. 2009.

LIMA E. C. F, FARIA M. D, MORAIS R. M. R. B, OLIVEIRA L. M. S. R, LIMA E. H. F, COSTA C. S. Interações entre meio ambiente, atendimentos antirrábicos e acidentes por animais peçonhentos no município de Petrolina(PE). Saúde Meio Ambient, v.6, n.1, p.54-70, jan./jun. 2017.

LIMA, C. A.; LEAL, A. L. R.; MANGUEIRA, S. A. L.; COSTA, S. M.; SANTOS, D. F. Vigilância em saúde: acidentes e óbitos provocados por animais peçonhentos na região sudeste Brasil, 2005-2015. Revista de Pesquisa: Cuidado é Fundamental Online, v. 12, p. 20-28, 2020.

LOPES A. B, OLIVEIRA A. A, DIAS F. C. F, SANTANA V. M. X, OLIVEIRA V. S, LIBERATO A. A, CALADO E. J. R, LOBO P. H. P, GUSMÃO K. E, GUEDES V. R. Perfil Epidemiológico dos Acidentes por Animais Peçonhentos na Região Norte Entre os Anos entre 2012 e 2015: Uma Revisão. Revista de Patologia do Tocantins, v.4, n.2, p.36-40, 2017.

NEIVA, C.A.C et al. Caracterização epidemiológica das intoxicações exógenas por substâncias nocivas e acidentes por animais peçonhentos em crianças no Estado do Amapá. Revista Científica Multidisciplinar Núcleo do Conhecimento, v. 01, p. 41-66, 2019. https://www.nucleodoconhecimento.com.br/saude/caracterizacaoepidemiologica

SALOMÃO, M. G.; LUNA, K. P. O.; MACHADO, C. Epidemiologia dos acidentes por animais peçonhentos e a distribuição de soros: estado de arte e a situação mundial. Rev. Salud Pública, v. 20, n. 4, p. 523-529, 2018.

$\mathrm{RC}: 67471$

Disponibile in: https://www.nucleodoconhecimento.com.br/salute/numero-di-infortuni 
SANTANA V. T. P, SUCHARA E. A. Epidemiologia dos acidentes com animais peçonhentos registrados em Nova Xavantina - MT. Revista de Epidemiologia e Controle de Infecção, v.5, n.3, p.141-146, 2015.

SILVA C. T., PARDAL P. P. Atividades Lúdicas Na Prevenção De Acidentes Por Animais Peçonhentos Em Estudantes Da Rede Pública Da Região Metropolitana De Belém. Revista Universo \& Extensão, v.3, n.3, 2015.

SILVA, A. M.; BERNARDE, P. S.; ABREU, L. C. Acidentes com animais peçonhentos no Brasil por sexo e idade. Journal of Human Growth and Development, v. 25, n. 1, p. 54-62, 2015a.

SILVA J. H, GIANSANTE S, SILVA R. C. R, SILVA G. B, SILVA L. B, PINHEIRO L. C. B. Perfil Epidemiológico dos Acidentes com Animais Peçonhentos em Tangará da Serra-Mt, Brasil (2007-2016). Journal Health NPEPS, p.5-15, 2017.

SOUZA C. M. V, MACHADO C. Animais peçonhentos de importância médica no município do rio de janeiro. Journal Health NPEPS, p.16-39 2017.

Presentato: Dicembre 2020.

Approvato: Dicembre 2020.

$\mathrm{RC}: 67471$

Disponibile in: https://www.nucleodoconhecimento.com.br/salute/numero-di-infortuni 